\title{
Editorial
}

\section{Evidence about the Chest CT diagnostic performance in the pandemic of SARS-CoV-2 infection: A smooth takeoff and hard landing.}

\author{
Dr Tamami Namiki \\ X並 木珠 並木 珠 \\ Women's Medical University Yachiyo Medical Center Tokyo Japan
}

According to all guidelines, the unique criterion for the diagnosis of severe acute respiratory syndrome coronavirus-2 (SARS-CoV-2) is the viral nucleic acid test by reverse transcription polymerase chain reaction (RT-PCR). That should always guide the isolation decision and the management conduction [1].

SARS-CoV-2 related pneumonia may manifest after 2 to 4 days following the coronavirus infection. Typical computed tomography (CT) feature of coronavirus (COVID-19) lung disease is the bilateral, peripheric or diffuse and patchy ground-glass opacities(GGO) with consolidations. Several other signs such as crazy paving pattern , interstitial thickening, reversed-halo or microvascular embolisms are less likely described and may evoke COVID-19 pneumonia in an appropriate context. The chest CT could be initially normal. The GGO have tendency of quick evolution till the seventh day and stabilize later till the $20^{\text {th }}$ day of the COVID-19 infection. Lung lesions in severe cases involve more lung segments with larger sized GGO. the interlobular septal thickening and consolidations are constant. The pleural effusion and pneumothorax were rarely described [2]. The lack of sensitivity, insufficient stability, and relatively long processing time of RT-PCR gave a considerable place for the chest CT in the daily practice of COVID-19 patients. Several reports found exciting the confrontation between the CT and the RT-PCR results. The sensitivity of CT features in highly pandemic areas could reach $97 \%$.

The earlier CT chest signs and the improvement of pulmonary lesions before RT-PCR negativity made some authors recommend the CT chest as diagnostic screening tool [3-5].

However, we still believe that the recommendation of the CT as a new diagnostic tool could not be based only on likelihood ratios and temporary concordance with the reference gold standard test.

The respect of the basic epidemiologic principles is always mandatory to enhance the conclusion's value. These recommendations were not born from blinded comparison with the refence test such in controlled randomized trials. To avoid time-lead bias many studies did not consider other potential bias especially the confusion and the selection. Moreover, the CT result did not influence the decision of proceeding to the reference test in all the previous reports. In this pandemic era, a chest CT examination will include not only additional cost for personal protective equipment (PPE) and sterilization but also more risk for the radiology department staff [6].

The chest CT could be used to select patients candidate for RT-PCR. Its value in the management and the follow up of pulmonary lesions cannot be overstated. It could play a role in clinical decision making when the gold standard test is not available in highly pandemic areas. Most of the radiology societies all over the world were not recommending the CT as diagnostic test especially in large scale screening. The few metaanalysis available have discordant conclusions about the topic. Upcoming evidence-based reports should contribute more to that $[7,8]$.

\section{References}

[1] Zhao W, Zhong Z, Xie X, Yu Q, Liu J. Relation Between Chest CT Findings and Clinical Conditions of Coronavirus Disease (COVID-19) Pneumonia: A Multicenter Study. Am J Roentgenol. 2020;21:1072-77.

[2] Sun Z, Zhang N, Li Y, Xu X. A systematic review of chest imaging findings in COVID-19. Quant Imaging Med Surg. 2020;10:1058-79.

[3] Sun Z. Diagnostic Value of Chest CT in Coronavirus Disease 2019 (COVID-19). Curr Med Imaging. 2020;16:274-275.

[4] Fang Y, Zhang H, Xie J, Lin M, Ying L, Pang P, et al. Sensitivity of Chest CT for COVID-19: Comparison to RT-PCR. Radiology. 2020;200432.

[5] Ai T, Yang Z, Hou H, Zhan C, Chen C, Lv W, et al. Correlation of Chest CT and RT-PCR Testing in Coronavirus Disease 2019 (COVID-19) in China: A Report of 1014 Cases. Radiology. 2020;200642.

[6] Nair A, Rodrigues JCL, Hare S, Edey A, Devaraj A, Jacob J, et al. A British Society of Thoracic Imaging statement: considerations in designing local imaging diagnostic algorithms for the COVID-19 pandemic. Clin Radiol. 2020;75:329-334.

[7] Xu B, Xing Y, Peng J, Zheng Z, Tang W, Sun Y, Xu C, Peng F. Chest CT for detecting COVID-19: a systematic review and meta-analysis of diagnostic accuracy. Eur Radiol. 2020:1-8.

[8] Bao C, Liu X, Zhang H, Li Y, Liu J. Coronavirus Disease 2019 (COVID-19) CT Findings: A Systematic Review and Meta-analysis. J Am Coll Radiol. 2020;17:701-9.

Citation: Namiki T. Evidence about the chest CT diagnostic performance in the pandemic of SARS-CoV-2 infection: A smooth takeoff and hard landing.Jr.med.res. 2020; 3(2):2. Namiki @). https://doi.org/10.32512/jmr.3.2.2020/2 